\title{
Effect of Ferrous ion on the performance of polycarboxylate superplasticizers
}

\author{
Shaofeng Wang ${ }^{1, ~ a}$, Long Xiong ${ }^{1, b}$, Juxiang Xing ${ }^{1, c}$, Guangjun Zheng $^{1}$, Yao Bi $^{1}$ \\ ${ }^{1}$ China Construction Ready Mixed Concrete Co. Ltd, Wuhan 430205, China \\ aemail: jaylia08@hotmail.com, bemail: xionglong1987@126.com, ${ }^{\mathrm{C}}$ email: xingjuxiang@126.com
}

Keywords: Ferrous ion; initiator; Molecular weight; dispersion properties

\begin{abstract}
The effect of ferrous ions on the molecular weight and molecular weight distribution of PCE is investigated by Gel permeation chromatography (GPC). The effect of ferrous ions on the dispersion properties of PCE is measured by the fluidity of cement pastes. The results indicate that ferrous sulfate adopted at the initiation system can accelerate the reaction and ensure the reaction rate at $5 \mathrm{C}-10 \mathrm{C}$. Ferrous sulfate dosage should be $0.02 \mathrm{wt} \%$ of the total monomers, which insures the best initial dispersibility and dispersion retaining ability of PCE.
\end{abstract}

\section{Introduction}

Due to a series advantages such as low dosage, high water reduction, small slump loss, environmental protection, Polycarboxylate superplasticizers have been widely used in water conservancy and hydropower engineering, road and rail, skyscraper [1-3]. In recent years under its synthesis process matures, new technologies emerging. While most of the synthesis process used at a high temperature in the range of 60-80C[4-6], which means large energy-consuming and pollution. An important method to reduce synthesis temperature is to adopt redox initiator system, which has attracted serious concern.

While as lowering reaction temperature, the reaction rate and the polymerization degree is changed accordingly, which also lead to affect the performance of Polycarboxylate. At present, low-temperature synthesis process of Polycarboxylate is still in the research stage. Current temperatures reported in the redox system mostly are at 15 30C[7-9]. Once the temperature is below this range, a series of bad phenomena will occur, such as low response rates, even not initiate the reaction.

The aim of this work is to add a small amount of ferrous ions in the conventional hydrogen peroxide / VC initiation system. Thereby, PCE synthesis initiation temperature dropped from $15 \mathrm{C}$ to $5 \mathrm{C}$, and the PCE demonstrated good performance.

\section{Experimental}

\subsection{Materials}

A Cement $42.5 \mathrm{R}$ was used supplied by huaxin Co., Ltd., which the specific surface area of 3500 $\mathrm{cm}^{2} / \mathrm{g}$. Acrylic acid used industrial raw materials from Qilu Petrochemical. TPEG 2400 Produced in Liaoning Occitan. Other chemicals were chemically pure (Aladdin).

\subsection{Synthesis of PCE}

The PCE was synthesized by aqueous free radical copolymerization. The appropriate concentration of hydrogen peroxide solution and TPEG-2400 were added to a 500ml four-necked flask as mass ratio of 1:1 ratio. A condenser, a nitrogen inlet and a stirring bar afterwards were equipped and give a homogeneous solution. When the solution starting temperature at $5 \mathrm{C}$, ice water bath was removed and four flask was placed in the air. Then acrylic acid, TGA, VC, ferrous sulfate, and water formulated as a solution and added to the flask under a fixed feeding rate. At the end, the $\mathrm{pH}$ was adjusted to 7 with $30 \mathrm{wt} \% \mathrm{NaOH}$ and the PCE was acquired. 


\subsection{Gel permeation chromatography (GPC)}

Molecular weight of PCE was measured by gel permeation chromatography (GPC, Waters 150C, USA), using the solution of methanol and buffer solution (1/2, v/v) as the eluent. (Column, PL aqua gel, $\mathrm{mix} / \mathrm{m}, 7.8 * 300 \mathrm{~mm}$, Agilent1100, Agilent Company, USA). The buffer solution was Na2B4O7/KCl aqueous solution $(\mathrm{pH}=8)$. Narrow polyethylene glycol (PEG) was used as calibration standards. The GPC detector included the refractive index.

\subsection{Mini slump test}

Test of the fluidity of cement pastes added with PCE was under the national standard GB 8077-2000 "methods for testing uniformity of concrete mixture" and the cement used for the test was HUAXIN P.O 42.5, the w/C ratio was 0.29 and the dosage of the PCE was $0.15 \%$ (by percent Weight based on solids cement).

\section{Experimental results and analysis}

\subsection{The effect of ferrous ions dosage on the molecular weight and molecular weight distribution of PCE}

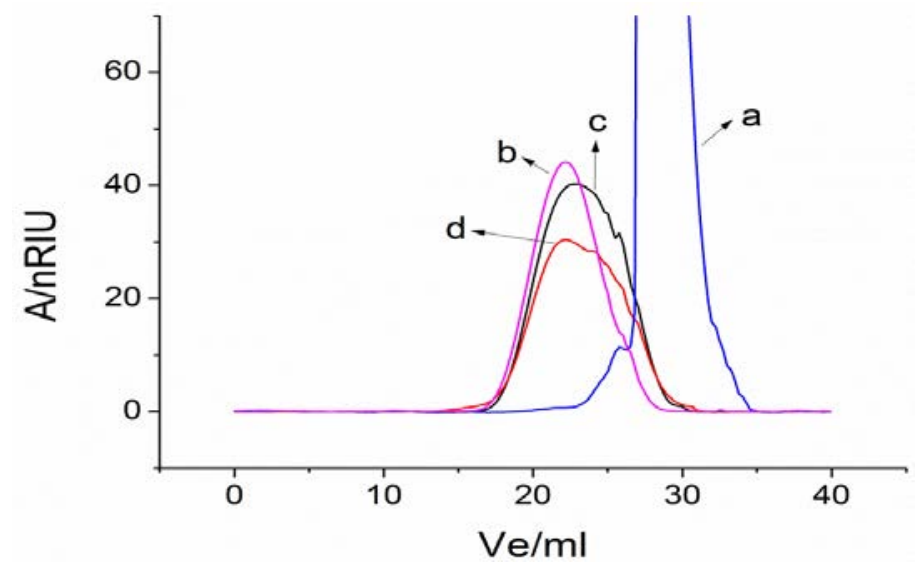

a is $0.00 \%$ ferrous sulfate of the total monomer mass $\mathrm{b}$ is $0.01 \%$ ferrous sulfate of the total monomer mass c is $0.02 \%$ ferrous sulfate of the total monomer mass $\mathrm{d}$ is $\mathbf{0 . 0 3 \%}$ ferrous sulfate of the total monomer mass

Fig. 1 Relationship between the different amount of ferrous ion and the molecular weight of PCE

As can be seen from the figure, when the polymerization reaction with hydrogen peroxide / VC initiator system and starting temperature was $5 \mathrm{C}$, the polymerization reaction rate is very slow. What's more, the product is almost no macromolecular substances and has a large number of residual monomer at the end of the reaction. However as $0.01 \%$ ferrous sulfate of the total mass of the monomer was adopted to the initiation system, the reaction rate was significantly faster. The results of GPC test showed no residual monomer, which meant monomer conversion rate has improved greatly. With the increase of the amount of ferrous sulfate, the molecular weight decreases and the molecular weight distribution broadens continuously.

Table 1 the calculated result of Fig. 1

$\begin{array}{cccc}\text { sample } & \mathrm{M}_{\mathrm{n}}(\mathrm{g} / \mathrm{mol}) & \mathrm{M}_{\mathrm{w}}(\mathrm{g} / \mathrm{mol}) & \mathrm{MWD} \\ 0.0 \% & 1256 & 2011 & 1.6 \\ 0.01 \% & 15133 & 43281 & 2.86 \\ 0.02 \% & 13128 & 39647 & 3.02 \\ 0.03 \% & 11534 & 37486 & 3.25\end{array}$




\subsection{The effect of the initial temperatures on the dispersion properties of PCE}

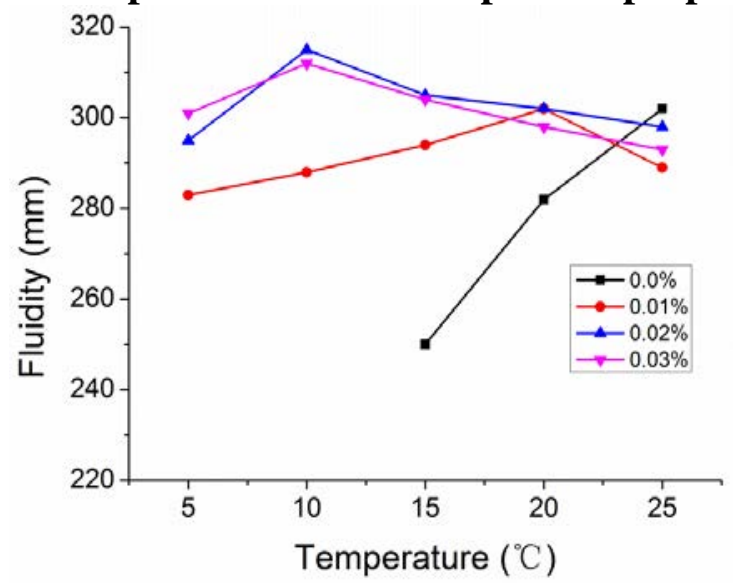

Fig. 2 Relationship between the initial temperatures and the dispersion properties of PCE

Figure 2 showed the effect of the initial temperatures on the dispersion properties of PCE. It was observed that as using only $\mathrm{H}_{2} \mathrm{O}_{2} / \mathrm{V}_{\mathrm{C}}$ redox initiator and the initial temperature was $5 \mathrm{C}$ or $10 \mathrm{C}$, PCE exhibited poor performance and its paste flows is too low and thus can not be measured. Whereas normal reaction could be proceed at the same initial temperature with just $0.01 \%$ ferrous sulfate of the total monomer mass. The initial cement paste fluidity was more than $280 \mathrm{~mm}$. As the increase of the amount of ferrous sulfate, paste fluidity increased slightly at low starting temperature and declined at a higher starting temperature. Therefore, ferrous sulfate dosage should be $0.02 \%$ based on the monomers total weight preferably.

\subsection{The effect of Ferrous sulfate content on the dispersion properties of PCE}

As can be seen from Figure 3 that the initial paste fluidity tended to increase with the increase in the amount of ferrous sulfate, but the slump performance actually was increased and then decreased. This may be due to the molecular weight decreases of PCE as the increased amount of ferrous sulfate. Lower molecular weight favoured initial absorption but was detriment of dispersion retaining ability.

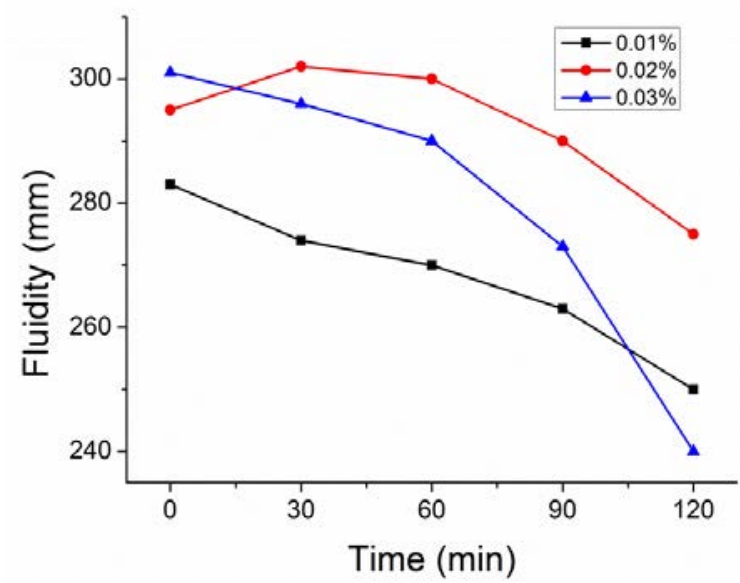

Fig. 3 Relationship between the Ferrous sulfate content and the dispersion properties of PCE

\section{Conclusions}

Conventional redox systems influenced by the activity of the unsaturated polyether monomer, when initial temperature is below $15 \mathrm{C}$, the polymerization reaction rate is too slow to get a good product's performance. When adding a trace amount of ferrous ions in the reaction system, the polymerization reaction rate is significantly increased, even the initial temperature is lowered to $5 \mathrm{C}$, the polymerization reaction can still be normal.GPC data indicate that the monomers are reacted completly, the polycarboxylate superplasticizers have a excellent performance. 


\section{References}

[1] Sakai E, Yamada K, Ohta A. Molecular Structure and Dispersion-Adsorption Mechanisms of Comb-Type Superplasticizers Used in Japan, Journal of Advanced Concrete Technology(2003), p16-25

[2] Yamada K, Takahashi T, Hanehara S, et al. Effects of the Chemical Structure on the Properties of Polycarboxylate-type Superplasticizer, Cement and Concrete Research(2000), p197-207

[3] D. Hamada T H M S. Development of New Superplasticizer Providing Ultimate Workability, Journal of ACI Special Publication(2006), p31-35

[4] Yoshioka K, Tazawa E, Kawai K, et al. Adsorption Characteristics of Superplasticizers on Cement Component Minerals, Cement and Concrete Research(2002), p1507-1513

[5] Yamada K. Basics of Analytical Methods Used for the Investigation of Interaction Mechanism between Cements and Superplasticizers, Cement and Concrete Research(2011), p793-798

[6] Zhang M, Sisomphon K, Ng T S, et al. Effect of Superplasticizers on Workability Retention and Initial Setting Time of Cement Pastes, Construction and Building Materials(2010), p1700-1707

[7] Zhuojun Jiang, Yunhui Fang, et al. Preparation of an extra high-concentration polycarboxylate superplasticizer, New Building Materials(2013), p29-31

[8] Guoxin Chen, Yeran Zhu, et al. Study on synthesis at room temperature and performance of slumpretention polycarboxylate superplasticizer, Concrete(2014), p68-69 